\title{
Toeplitz matrices in the Boundary Control method
}

\author{
M.I.Belishev, N.A.Karazeeva!
}

\begin{abstract}
Solving inverse problems by dynamical variant of the BC-method is basically reduced to inverting the connecting operator $C^{T}$ of the dynamical system, for which the problem is stated. Realizing the method numerically, one needs to invert the Gram matrix $\hat{C}^{T}=$ $\left\{\left(C^{T} f_{i}, f_{j}\right)\right\}_{i, j=1}^{N}$ for a representative set of controls $f_{i}$. To raise the accuracy of determination of the solution, one has to increase the size $N$, which, especially in the multidimensional case, leads to a rapid increase in the amount of computations. However, there is a way to reduce it by the proper choice of $f_{j}$, due to which the matrix $\hat{C}^{T}$ gets a specific block-Toeplitz structure. In the paper, we explain, where this property comes from, and outline a way to use it in numerical implementation of the BC-algorithms.
\end{abstract}

\section{Introduction}

- The BC-method (boundary control method) is an approach to inverse problems based on their connections with control theory [2]-6]. Its local variant, which is considered in the paper, directs to application in geophysics (seismology), where parameters of the medium depending on the depth must be determined from the data on the day surface. The wave process is initiated

*St. Petersburg Department of Steklov Mathematical Institute, St.Petersburg, Russia, e-mail: belishev@pdmi.ras.ru. Supported by the RFBR grant 18-01-00269 and VolksWagen Foundation.

${ }^{\dagger}$ St.Petersburg Department of Steklov Mathematical Institute, St. Petersburg, Russia, e-mail: karazeev@pdmi.ras.ru. 
by the sources (controls) disposed on a part of the boundary of the sounded domain; the data are recorded on the same part. In accordance with the finiteness of the wave propagation speed, the parameters must be determined in the real time: the longer is the observation time, the bigger is the depth of determination [2, 3, 4, 5].

Numerical algorithms based on the local variant are successfully tested in the series of experiments [2, 8, 7, 9, 15, 13, 16, 17, 18, 20]. Their further development and possible use in the work with real data is an important perspective goal. Our paper is adjacent to articles [10] and [11], which offer the simple tests for probing algorithms based on the BC-method. It reveals additional opportunities in numerical implementation of the BC-method.

- Typically, the inverse problems are nonlinear. The principal advantage of the classical approach by I.M.Gelfand, B.M.Levitan, M.G.Krein and V.A.Marchenko is that it reduces them to solving the linear problems and equations. The BC-method inherits this advantage. It reveals a unified view at the classical GLKM equations and shows that to solve them is to invert the so-called connecting operator $C^{T}$ of the dynamical system associated with the forward problem (see [12]). It is the fact, which provides the relevant multidimensional generalizations.

In accordance with these generalizations, to solve an inverse problem by dynamical variant of the BC-method is basically to invert the connecting operator $C^{T}$ of the relevant dynamical system. Respectively, realizing the method numerically, one needs to invert the Gram matrix $\hat{C}^{T}=$ $\left\{\left(C^{T} f_{i}, f_{j}\right)\right\}_{i, j=1}^{N}$ for a representative set of controls $f_{i}$, which simulates a basis in the space of controls. Along this way, we encounter two traditional obstacles:

1) to raise the accuracy of determination of the solution, one has to increase the number of controls (the size $N$ ), which, especially in the multidimensional case, leads to a rapid growth in the amount of computations;

2 ) as the size grows, the matrix $\hat{C}^{T}$ becomes more and more ill-conditional: the lower bound of its spectrum rapidly tends to zero.

The latter is unavoidable: it reflects the strong ill-posedness of multidimensional inverse problems, as well as of the corresponding boundary control problem, which is being solved within the BC-method. To deal with illposedness, a variety of regularization methods are used (see the works cited above). We pin certain hopes on A.A.Timonov's approach [18], which uses the mean curvature flow technique. 
- The given paper deals with the difficulty 1$)$. Its main subject is the remarkable fact that the matrix $\hat{C}^{T}$ may have a specific block-Toeplitz structure. Due to this, it is possible to significantly reduce the amount of computations during the determination of the inverse matrix $\left[\hat{C}^{T}\right]^{-1}$. We explain, where this property comes from, and outline a way to use it in numerical implementation of the BC-algorithms.

For the sake of determinacy and simplicity, we deal with a concrete inverse problem treated in [4] and [10, 11]. Namely, the simplicity is that the geometry of rays in the domain, which the problem is considered in, is Euclidean. However, the Toeplitz structure of $\hat{C}^{T}$ is the fact of rather general character.

- The authors thank I.V.Kubyshkin for the kind help in computer graphics.

\section{Toeplitz structure}

\section{Forward problems}

- The initial boundary value problem

$$
\begin{array}{ll}
u_{t t}-\Delta u-\langle\nabla \ln \rho, \nabla u\rangle=0 & \text { in } \mathbb{R}_{+}^{2} \times(0, T), \\
\left.u\right|_{t=0}=\left.u_{t}\right|_{t=0}=0 & \text { in } \overline{\mathbb{R}_{+}^{2}}, \\
\left.u_{y}\right|_{y=0}=f & \text { for } 0 \leqslant t \leqslant T,
\end{array}
$$

is considered in the half-plain $\mathbb{R}_{+}^{2}:=\left\{(x, y) \in \mathbb{R}^{2} \mid y>0\right\}$, where $\Delta u=$ $u_{x x}+u_{y y},\langle\nabla \ln \rho, \nabla u\rangle=(\ln \rho)_{x} u_{x}+(\ln \rho)_{y} u_{y}, \rho=\rho(x, y)$ is a smooth positive function (reduced sound velocity), $f=f(x, t)$ is a Neumann boundary control, $T>0$ is a final time, $u=u^{f}(x, y, t)$ is a solution (wave).

- The solutions to the hyperbolic problem (1)-(3) obey a finiteness of the domain of influence principle. Let $\sigma=\{(x, 0) \mid \alpha \leqslant x \leqslant \beta\}$ be a finite segment of the boundary $\mathbb{R}_{x}:=\partial \mathbb{R}_{+}^{2}$,

$$
\Omega_{\sigma}^{r}:=\left\{p \in \mathbb{R}_{+}^{2} \mid \operatorname{dist}(p, \sigma)<r\right\}
$$

its metric neighborhood of radius $r>0$ (dist is the Euclidean distance in $\left.\overline{\mathbb{R}_{+}^{2}}\right)$. If $\operatorname{supp} f \subset \bar{\sigma} \times[0, T]$, i.e., the control is supported on the segment $\sigma$, then

$$
\operatorname{supp} u^{f}(\cdot, \cdot, t) \subset \Omega_{\sigma}^{t}, \quad 0<t \leqslant T
$$


holds, i.e., the corresponding wave is localized in the $t$-neighborhood of the segment, from which the control acts.

Relation (4) means that the waves propagate in a half-plane with the unit velocity.

- By hyperbolicity, for the controls $f$ provided supp $f \subset \bar{\sigma} \times[0,2 T]$, the following extended problem is also well posed:

$$
\begin{array}{ll}
u_{t t}-\Delta u-\langle\nabla \ln \rho, \nabla u\rangle=0, & p \in \Omega_{\sigma}^{T}, 0<t<2 T-\operatorname{dist}(p, \sigma) ; \\
u=0 & \text { for } t<\operatorname{dist}(p, \sigma) ; \\
\left.u_{y}\right|_{y=0}=f & \text { for } 0 \leqslant t \leqslant 2 T,
\end{array}
$$

where $p=(x, y) \in \mathbb{R}_{+}^{2}$.

\section{Dynamical system}

Problem (1)-(3) is endowed with standard attributes of a dynamical system: spaces and operators.

- An outer space is the space $\mathscr{F}_{\sigma}^{T}:=L_{2}(\sigma \times[0, T])$ of controls acting from $\sigma$, with the inner product

$$
(f, g)_{\mathscr{F}_{\sigma}^{T}}=\int_{\sigma \times[0, T]} f(x, t) g(x, t) \rho(x, 0) d x d t .
$$

- An inner space of the states (waves) is $\mathscr{H}_{\sigma}^{T}:=L_{2, \rho}\left(\Omega_{\sigma}^{T}\right)$ with the inner product

$$
(v, w)_{\mathscr{H}_{\sigma}^{T}}=\int_{\Omega_{\sigma}^{T}} v(x, y) w(x, y) \rho(x, y) d x d y .
$$

By virtue of (4) the waves $u^{f}(\cdot, \cdot, t)$ are its elements (the functions of the variables $x$ and $y$ depending on $t$ as a parameter).

- A control operator $W^{T}: \mathscr{F}_{\sigma}^{T} \rightarrow \mathscr{H}_{\sigma}^{T}$,

$$
\left(W^{T} f\right)(x, y):=u^{f}(x, y, T), \quad(x, y) \in \mathbb{R}_{+}^{2}
$$

is the operator, which solves problem (1)-(3). It is compact and injective for any $T>0$ [4].

- A response operator $R^{T}: \mathscr{F}_{\sigma}^{T} \rightarrow \mathscr{F}_{\sigma}^{T}$,

$$
\left(R^{T} f\right)(x, t):=u^{f}(x, 0, t), \quad x \in \sigma, 0 \leqslant t \leqslant T
$$


describes the reaction of the system on the effect of control. It is also compact. The right-hand side in this definition means a pressure observed on the boundary of the half-plane. The well-known representation

$$
\left(R^{T} f\right)(x, t):=\int_{0}^{t} d s \int_{\sigma} r\left(x, x^{\prime} ; t-s\right) f\left(x^{\prime}, s\right) d x^{\prime}, \quad x \in \sigma, \quad 0 \leqslant t \leqslant T
$$

holds with a piece-wise continuous kernel $r$.

- An extended response operator $R^{2 T}: \mathscr{F}_{\sigma}^{2 T} \rightarrow \mathscr{F}_{\sigma}^{2 T}$,

$$
\left(R^{2 T} f\right)(x, t):=u^{f}(x, 0, t), \quad x \in \sigma, 0 \leqslant t \leqslant 2 T
$$

is associated with the problem (5)-(7) and the representation

$$
\left(R^{2 T} f\right)(x, t):=\int_{0}^{t} d s \int_{\sigma} r\left(x, x^{\prime} ; t-s\right) f\left(x^{\prime}, s\right) d x^{\prime}, \quad x \in \sigma, \quad 0 \leqslant t \leqslant 2 T
$$

holds. Both operators $R^{T}$ and $R^{2 T}$ are compact. Also, both of them are determined by the values of the function $\rho$ in $\Omega_{\sigma}^{T}$ only (do not depend on $\rho$ outside $\Omega_{\sigma}^{T}$ ).

- An operator $C^{T}: \mathscr{F}_{\sigma}^{T} \rightarrow \mathscr{F}_{\sigma}^{T}$,

$$
C^{T}:=\left(W^{T}\right)^{*} W^{T}
$$

is called connecting operator. For controls $f, g \in \mathscr{F}_{\sigma}^{T}$ one has

$$
\left(u^{f}(\cdot, \cdot, T), u^{g}(\cdot, \cdot, T)\right)_{\mathscr{H}_{\sigma}^{T}} \stackrel{\underline{9}}{=}\left(W^{T} f, W^{T} g\right)_{\mathscr{H}_{\sigma}^{T}}=\left(C^{T} f, g\right)_{\mathscr{F}_{\sigma}^{T}} ;
$$

so $C^{T}$ connects the metrics of the outer and inner spaces. In view of compactness and injectivity of $W^{T}$, the operator $C^{T}$ is also compact and injective. Also, by its definition (11), $C^{T}$ is self-adjoint and positive.

- One of the key facts of BC-method is a simple and explicit relation between the response and connecting operators (see [2, 3, 4]). For its formulation we introduce:

the operator of the odd extension $S^{T}: \mathscr{F}_{\sigma}^{T} \rightarrow \mathscr{F}_{\sigma}^{2 T}$,

$$
\left(S^{T} f\right)(\cdot, t):= \begin{cases}f(\cdot, t), & 0 \leqslant t<T \\ -f(\cdot, 2 T-t), & T \leqslant t \leqslant 2 T\end{cases}
$$


the integration $J^{2 T}: \mathscr{F}_{\sigma}^{2 T} \rightarrow \mathscr{F}_{\sigma}^{2 T}$

$$
\left(J^{2 T} f\right)(\cdot, t):=\int_{0}^{t} f(\cdot, s) d s, \quad 0 \leqslant t \leqslant 2 T
$$

the operator $P^{2 T}: \mathscr{F}_{\sigma}^{2 T} \rightarrow \mathscr{F}_{\sigma}^{2 T}$ that selects the odd part of 'long' controls:

$$
\left(P^{2 T} f\right)(\cdot, t):=\frac{1}{2}[f(\cdot, t)-f(\cdot, 2 T-t)], \quad 0 \leqslant t \leqslant 2 T
$$

the reduction operator $N^{2 T}: \mathscr{F}_{\sigma}^{2 T} \rightarrow \mathscr{F}_{\sigma}^{T}$,

$$
\left(N^{2 T} f\right)(\cdot, t):=f(\cdot, t), \quad 0 \leqslant t \leqslant T .
$$

Also, note a simply verified relation $\left(S^{T}\right)^{*}=2 N^{2 T} P^{2 T}$.

Lemma 1. The representation

$$
C^{T}=\frac{1}{2}\left(S^{T}\right)^{*} J^{2 T} R^{2 T} S^{T}
$$

is valid.

\section{Inverse problem}

In the domain $\Omega_{\sigma}^{T}$ filled with waves at the final moment $t=T$, one selects a subdomain (ray tube)

$$
B_{\sigma}^{T}:=\{(x, y) \mid x \in \sigma, 0 \leqslant y<T\}
$$

which is covered by the rays emanated from the points of $\sigma$ orthogonally to the boundary (see Fig.1).

The inverse problem is to determine the function $\rho$ in the tube $B_{\sigma}^{T}$ from the given extended response operator $R^{2 T}$. Also, for the sake of simplicity, we assume that the function $\rho(\cdot, 0)$ is known in $\sigma$ that enables one to use the product 8 . 




Fig. 1 Domain $\mathrm{B}_{\sigma}^{\mathrm{T}} \subset \Omega_{\sigma}^{\mathrm{T}}$ 


\section{BCP}

- As usual in the BC-method, one relates the inverse problem with a corresponding boundary control problem (BCP). For the problem stated above, the relevant $\mathrm{BCP}$ is the following.

Let $1_{\sigma}^{T} \in \mathscr{H}_{\sigma}^{T}$ be the function equal to 1 identically in $\Omega_{\sigma}^{T}$. The BCP is to find the control $f \in \mathscr{F}_{\sigma}^{T}$, which provides

$$
u^{f}(\cdot, \cdot, T)=1_{\sigma}^{T} .
$$

To be more precise, for determination of $\left.\rho\right|_{B_{\sigma}^{T}}$ one needs to solve a family of the 'shortened' problems 13 with the r.h.s. $1_{\sigma}^{\xi} \in \mathscr{H}_{\sigma}^{\xi}$ for all $0<\xi \leqslant T$ and then apply the so-called amplitude formula [4, 10]. However, to demonstrate the appearance of Toeplitz matrices, it suffices to consider a single problem (13).

- Writing (13) in the form $W^{T} f=1_{\sigma}^{T}$ (see (9) ) and applying the adjoint operator, we get $\left(W^{T}\right)^{*} W^{T} f=\left(W^{T}\right)^{*} 1_{\sigma}^{T}$. Integration by parts provides $\left(W^{T}\right)^{*} 1_{\sigma}^{T}=\varkappa^{T}$, where $\varkappa^{T} \in \underset{\sigma}{\mathscr{F}_{\sigma}^{T}}: \varkappa^{T}(x, t):=T-t$ (see [4]). At last, recalling the definition of the connecting operator, we arrive at the equation

$$
C^{T} f=\varkappa^{T} \quad \text { in } \mathscr{F}_{\sigma}^{T} .
$$

For the subsequent, it is convenient to reduce 114 to an equation in $\mathscr{F}_{\sigma}^{2 T}=$ $L_{2}([0,2 T] \times \sigma)$ as follows.

Denote $\tilde{f}:=S^{T} f \in \mathscr{F}_{\sigma}^{2 T}$ and $p\left(x, x^{\prime} ; t\right):=\int_{0}^{t} r\left(x, x^{\prime} ; s\right) d s$.

Lemma 2. Equation (14) is equivalent to the equation in $\mathscr{F}_{\sigma}^{2 T}$ of the form

$$
\int_{0}^{2 T} d s \int_{\sigma} p\left(x, x^{\prime} ;|t-s|\right) \tilde{f}\left(x^{\prime}, s\right) d x^{\prime}=4(T-t), \quad(x, t) \in \sigma \times[0,2 T] .
$$

Proof. By 10, the operator $M^{2 T}:=J^{2 T} R^{2 T}$ acts in $\mathscr{F}_{\sigma}^{2 T}$ by the rule

$$
\begin{aligned}
& \left(M^{2 T} f\right)(x, t)=\int_{0}^{t} d s \int_{\sigma} p\left(x, x^{\prime} ; t-s\right) f\left(x^{\prime}, s\right) d x^{\prime}= \\
& =\int_{0}^{t} d s \int_{\sigma} p\left(x, x^{\prime} ;|t-s|\right) f\left(x^{\prime}, s\right) d x^{\prime}, \quad(x, t) \in \sigma \times[0,2 T] .
\end{aligned}
$$


Its adjoint acts by

$$
\begin{aligned}
& \left(\left(M^{2 T}\right)^{*} f\right)(x, t)=\int_{t}^{2 T} d s \int_{\sigma} p\left(x, x^{\prime} ; s-t\right) f\left(x^{\prime}, s\right) d x^{\prime}= \\
& =\int_{t}^{2 T} d s \int_{\sigma} p\left(x, x^{\prime} ;|s-t|\right) f\left(x^{\prime}, s\right) d x^{\prime}, \quad(x, t) \in \sigma \times[0,2 T] .
\end{aligned}
$$

As a result, we have

$$
\begin{aligned}
& \left(\left[M^{2 T}+\left(M^{2 T}\right)^{*}\right] f\right)(x, t)= \\
& =\int_{0}^{2 T} d s \int_{\sigma} p\left(x, x^{\prime} ;|t-s|\right) f\left(x^{\prime}, s\right) d x^{\prime}, \quad(x, t) \in \sigma \times[0,2 T] .
\end{aligned}
$$

In the meantime, 12 implies

$$
\begin{aligned}
& C^{T}=\left(C^{T}\right)^{*}=\frac{1}{2}\left[C^{T}+\left(C^{T}\right)^{*}\right]= \\
& =\frac{1}{2}\left[\frac{1}{2}\left(S^{T}\right)^{*} J^{2 T} R^{2 T} S^{T}+\frac{1}{2}\left(S^{T}\right)^{*}\left(J^{2 T} R^{2 T}\right)^{*} S^{T}\right]= \\
& =\frac{1}{4}\left(S^{T}\right)^{*}\left[M^{2 T}+\left(M^{2 T}\right)^{*}\right] S^{T} .
\end{aligned}
$$

Applying $S^{T}$ to 14 and using $S^{T}\left(S^{T}\right)^{*}=I$, we arrive at

$$
\left[M^{2 T}+\left(M^{2 T}\right)^{*}\right] \tilde{f}=4 \tilde{\varkappa}^{T} \quad \text { in } \mathscr{F}_{\sigma}^{2 T},
$$

where $\tilde{f}:=S^{T} f, \tilde{\varkappa}^{T}:=S^{T} \varkappa^{T}=T-t$. Then 16 implies 15.

To get the solution $f$ to 14 , one can solve 15 and then take $f=\left.\tilde{f}\right|_{0 \leqslant t \leqslant T}$.

Equations (14) and (15) are the relevant multidimensional analogs of the classical GLKM equations: see [1, 12].

\section{Toeplitz matrix}

- Solving 15 numerically, the solution $\tilde{f}$ is sought in the form of an expansion over a complete linearly independent system of controls in $\mathscr{F}_{\sigma}^{2 T}$. Such 
a system is simulated by a finite sufficiently rich system of controls, to the description of which we proceed.

In the space-time plane $\mathbb{R}_{(x, t)}^{2}$, choose a function (basic source)

$$
g=g(x, t): \quad \operatorname{supp} g=\Delta_{0}^{0}:=[0, \varepsilon] \times[0, \delta] \quad(\varepsilon, \delta>0)
$$

supported on the basic rectangular $\Delta_{0}^{0}$; let

$\Delta_{j}^{i}:=\left\{(x+j \varepsilon, t+i \delta) \mid(x, t) \in \Delta_{0}^{0}, \quad j=0,1, \ldots, M-1 ; i=0,1, \ldots, N-1\right\}$

(see Fig.2) and $g_{j}^{i}(x, t):=g(x-j \varepsilon, t-i \delta), g_{0}^{0}:=g$. For the fixed (big) $M$ and $N$, fit $\varepsilon=|\sigma| / M, \delta=(2 T) / N$ to provide the partition

$$
\sigma \times[0,2 T]=\bigcup_{\substack{j=0,1, \ldots, M-1 ; \\ i=0,1, \ldots, N-1}} \Delta_{j}^{i} .
$$

Take $g_{j}^{i}=g_{j}^{i}(x, t):=g(x-j \varepsilon, t-i \delta)$ supported in $\Delta_{j}^{i}$. By their choice, the controls $g_{j}^{i}$ are linearly independent.



\section{Fig. 2 Basis of controls}

- Solving (15), we seek for the solution in the form

$$
\tilde{f}(x, t)=\sum_{\substack{j=0,1, \ldots, M-1 ; \\ i=0,1, \ldots, N-1}} c_{j}^{i} g_{j}^{i}(x, t)
$$


with the unknown $c_{j}^{i}$ and arrive at the linear system

$$
\sum_{\substack{j=0,1, \ldots, M-1 ; \\ i=0,1, \ldots, N-1}} G_{j l}^{i k} c_{j}^{i}=4\left(\tilde{\varkappa}^{T}, g_{l}^{k}\right)_{\mathscr{F}_{\sigma}^{2 T}}, \quad j, l=1, \ldots, M-1 ; i, k=1, \ldots, N-1
$$

with the Gram matrix

$$
\begin{aligned}
G_{j l}^{i k}:=\left(\left[M^{2 T}+\left(M^{2 T}\right)^{*}\right] g_{j}^{i}, g_{l}^{k}\right)_{\mathscr{F}^{2 T}} \stackrel{15}{=} \\
=\int_{\Delta_{l}^{k}} d x d t g_{l}^{k}(x, t) \int_{\Delta_{j}^{i}} p\left(x, x^{\prime} ;|t-s|\right) \tilde{g}_{j}^{i}\left(x^{\prime}, s\right) d s d x^{\prime}= \\
=\int_{\Delta_{l}^{k}} d x d t g(x-l \varepsilon, t-k \delta) \int_{\Delta_{j}^{i}} p\left(x, x^{\prime} ;|t-s|\right) \tilde{g}\left(x^{\prime}-\varepsilon j, s-i \delta\right) d s d x^{\prime}= \\
=\int_{\Delta_{0}^{0}} d x d t g(x, t) \int_{\Delta_{0}^{0}} p\left(x+l \varepsilon, x^{\prime}+\varepsilon j ;|t-k \delta-(s-i \delta)|\right) \tilde{g}\left(x^{\prime}, s\right) d s d x^{\prime}= \\
=\int_{\Delta_{0}^{0}} d x d t g(x, t) \int_{\Delta_{0}^{0}} p\left(x+l \varepsilon, x^{\prime}+\varepsilon j ;|t-s+(k-i) \delta|\right) \tilde{g}\left(x^{\prime}, s\right) d s d x .
\end{aligned}
$$

Its entries depend on the difference $k-i$, so that we have a block-Töplitz matrix with $N \times N$ blocks of the size $M \times M$. Such a peculiarity of the Gram system is due to the proper choice of the controls $g_{j}^{i}$. For the first time, it was used in numerical testing by V.Yu.Gotlib in [8, 7].

The r.h.s. of the system $(17)$ is

$4\left(\tilde{\varkappa}^{T}, g_{l}^{k}\right)_{\mathscr{F}_{\sigma}^{2 T}}=4 \int_{\sigma \times[0,2 T]}(T-t) g_{l}^{k}(x, t) d x d t=4 \int_{\Delta_{l}^{k}}(T-t) g_{l}^{k}(x, t) d x d t=: \beta_{l}^{k}$.

Thus, the system is fully determined by the inverse data $R^{2 T}$ and the choice of controls $g_{j}^{i}$.

\section{Inverting algorithm}

The algorithms provided in [19] are apropriate for a wide class of nonsingular block-Toeplitz matrices. However, the Gram matrix $G_{j l}^{i k}$, with which we deal, 
is not only block-Toeplitz but also symmetric and positive. This is the case we are considering in this section.

- So, we deal with a nonsingular symmetric positive block-Toeplitz matrix

$$
\mathbf{G}=\left\{G_{j l}^{i k}\right\}_{\substack{j, l=0,1, \ldots, M-1 ; \\
i, k=0,1, \ldots, N-1}}=\left(\begin{array}{ccccc}
\gamma_{0} & \gamma_{1} & \gamma_{2} & \ldots & \gamma_{N-1} \\
\gamma_{1} & \gamma_{0} & \gamma_{1} & \ldots & \gamma_{N-2} \\
\gamma_{2} & \gamma_{1} & \gamma_{0} & \ldots & \gamma_{N-3} \\
\vdots & \vdots & \vdots & \ddots & \vdots \\
\gamma_{N-1} & \gamma_{N-2} & \gamma_{N-3} & \ldots & \gamma_{0}
\end{array}\right)
$$

where the blocks $\gamma_{m}$ are the $M \times M$ - matrices with the entries $G_{j l}^{i k}$, $|i-k|=m ; m=0, \ldots, N-1$. The indices $j$ and $l$ take the values $0 \leqslant j, l \leqslant$ $M-1$.

Introduce the row of the unknowns

$$
\mathscr{C}:=\left(c_{0}^{0}, \ldots, c_{M-1}^{0} ; c_{0}^{1}, \ldots, c_{M-1}^{1} ; \ldots ; c_{0}^{N-1}, \ldots, c_{M-1}^{N-1}\right)
$$

and the row of the r.h.s. $\beta_{l}^{k}$

$$
\mathscr{B}:=\left(\beta_{0}^{0}, \ldots, \beta_{M-1}^{0} ; \beta_{0}^{1}, \ldots, \beta_{M-1}^{1} ; \ldots ; \beta_{0}^{N-1}, \ldots, \beta_{M-1}^{N-1}\right)
$$

defined by (18), the rows being of the length $M N$. Then (17) is equivalent to the system

$$
\mathscr{C} \mathbf{G}=\mathscr{B}
$$

To solve (19) one needs to invert the matrix G. For this, we apply a Levinson type algorithm presented in [19]. The construction of the inverse matrix relies on the Theorem 1 taken from [19] and exposed below.

In what follows, the accent ' denotes the operation of the block transposition. That is, the blocks are transposed but their inner structure is not disturbed.

Let $Y=\left(Y_{0}, \ldots, Y_{N-1}\right)^{\prime}$ be the block column, which satisfies the relation

$$
\mathrm{G} Y=\mathscr{I}_{N}=\left(\begin{array}{c}
O \\
O \\
\vdots \\
I
\end{array}\right)
$$

where $I$ and $O$ are the unit and zero $M \times M$ - matrices. Such $Y$ does exist and is unique just because $\mathbf{G}$ is nonsingular. The block column $\mathscr{I}_{N}$ is the 
matrix consisting of $M N$ rows and $M$ columns; $Y$ is also a matrix of the same size. Moreover, as is shown in [19], the $M \times M$ - matrix $Y_{N-1}$ is necessarily nonsingular.

Theorem 1. Let $Y$ be determined by (20). Then the representation

$$
\begin{gathered}
\mathbf{G}^{-1}=\left(\begin{array}{cccc}
Y_{N-1} & O & \ldots & O \\
Y_{N-2} & Y_{N-1} & \ldots & O \\
\vdots & \vdots & \ddots & \vdots \\
Y_{0} & Y_{1} & \ldots & Y_{N-1}
\end{array}\right) Y_{N-1}^{-1}\left(\begin{array}{ccccc}
Y_{N-1} & Y_{N-2} & \ldots & Y_{0} \\
O & Y_{N-1} & \ldots & Y_{1} \\
\vdots & \vdots & \ddots & \vdots \\
O & O & \ldots & Y_{N-1}
\end{array}\right)- \\
-\left(\begin{array}{cccc}
O & O & \ldots & O \\
Y_{0} & O & \ldots & O \\
\vdots & \vdots & \ddots & \vdots \\
Y_{N-2} & Y_{N-3} & \ldots & O
\end{array}\right) Y_{N-1}^{-1}\left(\begin{array}{cccc}
O & Y_{0} & \ldots & Y_{N-2} \\
O & O & \ldots & Y_{N-3} \\
\vdots & \vdots & \ddots & \vdots \\
O & O & \ldots & O
\end{array}\right)
\end{gathered}
$$

is valid.

- By positivity of $\mathbf{G}$, all the matrices

$$
\mathbf{G}_{k}=\left(\begin{array}{cccc}
\gamma_{0} & \gamma_{1} & \ldots & \gamma_{k} \\
\gamma_{1} & \gamma_{0} & \ldots & \gamma_{k-1} \\
\vdots & \vdots & \ddots & \vdots \\
\gamma_{k} & \gamma_{k-1} & \ldots & \gamma_{0}
\end{array}\right)
$$

of the order $k+1(k=0, \ldots, N-1)$ are symmetric and positive definite. In particular, $\gamma_{0}$ is positive definite and, hence, invertible.

To find the block column $Y$ one can use the following recurrent Procedure. Let

$$
Y^{(k)}=\left(Y_{0}^{(k)}, Y_{1}^{(k)}, \ldots, Y_{k}^{(k)}\right)^{\prime}
$$

be found at the previous steps. So, $Y^{(k)}$ consists of $k+1$ blocks of the size $M \times M$ and satisfies the system (20) of the size $k+1(0 \leqslant k \leqslant N-1)$.

The Levinson algorithm [19], which we apply, makes the use of the normalizing factors $Q_{k}(M \times M$-matrix $)$ entering in the representation

$$
Y_{l}^{(k)}=\tilde{Y}_{l}^{(k)} Q_{k} ; \quad l=0, \ldots, k \quad(k=0,1, \ldots, N-1)
$$

and being also determined during the Procedure.

- The Procedure is as follows. 
1. At the first step we determine $\tilde{Y}_{0}^{(0)}=\gamma_{0}^{-1} Q_{0}^{-1}$, where $Q_{0}$ is arbitrary nonsingular $M \times M$-matrix. In particular one can take $Q_{0}=I$.

2. Let the block vector $\tilde{Y}^{(k-1)}$ and factor $Q_{k-1}$ be already found. Introduce the auxiliary matrices $E_{k}$ and $F_{k}$ by

$$
E_{k}=\gamma_{1} \tilde{Y}_{0}^{(k-1)}+\gamma_{2} \tilde{Y}_{1}^{(k-1)}+\ldots+\gamma_{k} \tilde{Y}_{k-1}^{(k-1)}, \quad F_{k}=-Q_{k-1} E_{k}
$$

whereas the matrix $I-F_{k}^{2}$ turns out to be nonsingular [19]. Then the factor $Q_{k}$ is determined by

$$
Q_{k}=\left(I-F_{k}^{2}\right)^{-1} Q_{k-1}
$$

3. At the next step one computes the block vector $\tilde{Y}^{(k)}$ by

$$
\begin{aligned}
& \left(\tilde{Y}_{0}^{(k)}, \tilde{Y}_{1}^{(k)}, \ldots, \tilde{Y}_{k}^{(k)}\right)^{\prime}=\left(\tilde{Y}_{k-1}^{(k-1)}, \tilde{Y}_{k-2}^{(k-1)}, \ldots, \tilde{Y}_{0}^{(k-1)}, 0\right)^{\prime} F_{k}+ \\
& +\left(0, \tilde{Y}_{0}^{(k-1)}, \tilde{Y}_{1}^{(k-1)}, \ldots, \tilde{Y}_{k-1}^{(k-1)}\right)^{\prime}
\end{aligned}
$$

and determines

$$
Y_{l}^{(k)}=\tilde{Y}_{l}^{(k)} Q_{k} ; \quad l=0, \ldots, k .
$$

4. Proceeding up to $k=N-1$, we find the block column $Y^{(N-1)}=Y$. Then, by the use of (21), we get the matrix $\mathbf{G}^{-\mathbf{1}}$.

The algorithm requires $\sim M^{3} N^{2}$ operations and, thus, may be regarded as "fast" [19].

Inversion of Toeplitz matrices is also treated in the paper [14. However, it does not deal with the block matrices, as is necessary in multidimensional

problems. In the meantime, perhaps, the algorithms of [14] may be developed for this case.

\section{References}

[1] M.I.Belishev. The Gelfand-Levitan type equations in multidimensional inverse problem for the wave equation. Zap. Nauchn. Semin. LOMI, 165, 15-20 (Engl. transl. 1990 J. Sov. Math., 1990, v.50, No 6, P. 1940-1944). 
[2] M.I.Belishev. Boundary control in reconstruction of manifolds and metrics (the BC method). Inverse Problems, 13, No. 5 (1997), R1-R45.

[3] M.I.Belishev. How to see waves under the Earth surface (the BC-method for geophysicists). Ill-Posed and Inverse Problems. S. I. Kabanikhin and V. G. Romanov (Eds). VSP, 2002, pp. 55-72.

[4] M.I.Belishev. Dynamical Inverse Problem for the Equation $u_{t t}-\Delta u-$ $\nabla \rho \cdot \nabla u=0$ (the BC-Method). CUBO A Math. J. 10, No, 2 (2008), $17-33$.

[5] M.I.Belishev. Boundary Control Method. Encyclopedia of Applied and Computational Mathematics, Vol. 1, pp. 142-146.

[6] M.I.Belishev. Boundary control and tomography of Riemannian manifolds (BC-method). Uspechi Mat. Nauk 72 (2017), No. 4, 3-66 (in Russian).

English translation: M.I.Belishev. Boundary control and tomography of Riemannian manifolds (the BC-method). Russian Math. Surveys; DOI 10.1070/RM9768.

[7] M.I.Belishev, V.Yu.Gotlib. Dynamical variant of the BC-method: theory and numerical testing. J. Inverse and Ill-Posed Problems 7, No. 3 (1999), 221-240.

[8] M.I.Belishev, V.Yu.Gotlib, S.A.Ivanov. The BC-method in multidimensional spectral inverse problem: theory and numerical illustrations. Control, Optimization and Calculus of Variations, 2 (1997), October, 307327.

[9] M.I.Belishev, I. B. Ivanov, I. V. Kubyshkin, V. S. Semenov. Numerical testing in determination of sound speed from a part of boundary by the BC-method. J. Inverse and Ill-Posed Problems 24 (2016), Issue 2, 159180. DOI: $10.1515 /$ jiip-2015-0052.

[10] M.I.Belishev, N.A.Karazeeva. Simplest Test for the Two-Dimensional Dynamical Inverse Problem (BC-Method). J Math Sci, (2019). https://doi.org/10.1007/s10958-019-04567-5. 
[11] M.I.Belishev, A.S.Blagoveshchensky, N.A.Karazeeva. Simplest Test for the Three-Dimensional Dynamical Inverse Problem (The BC-Method). J Math Sci (2021). https://doi.org/10.1007/s10958-021-05182-z.

[12] M.I.Belishev, V.S.Mikhailov Unified approach to classical equations of inverse problem theory. Journal of Inverse and Ill-Posed Problems, 20 (2012), no 4, 461-488.

[13] I.B.Ivanov, M.I.Belishev, V.S.Semenov. The reconstruction of sound speed in the Marmousi model by the boundary control method. arXiv: 1609.07586v1 [physics.geo-ph] 24 Sept 2016.

[14] G.Heinig, K.Rost. Fast algorithms for Toeplitz and Hankel matrices. Linear Algebra and its Applications, 435 (2011, 1-59. doi:10.1016/j.laa.2010.12.001.

[15] M.V.De Hoop, P.Kepley, L.Oksanen. Recovery of a smooth metric via wave field and coordinate transformation reconstruction. SIAM J. Appl. Math. 78, No. 4 (2018), 1931-1953.

[16] L.Oksanen. Solving an inverse obstacle problem for the wave equation by using the boundary control method. Inverse Problems 29 (2013), No. 3, 035004; doi:10.1088/0266-5611/29/3/035004.

[17] L.Pestov, V.Bolgova and O.Kazarina. Numerical recovering of a density by the BC-method. Inverse Problems and Imaging, Volume 4, No. 4, 2010, 703-712. doi:10.3934/ipi.2010.4.703.

[18] A.A.Timonov. A novel method for the numerical solution of a hybrid inverse problem of electrical conductivity imaging. Zapiski Nauch. Semin. POMI, 499 (2020), 105-128 (in Russian).

[19] V.V. Voevodin, E.E. Tyrtyshnikov. Numerical calculus of Toeplitz matrices. Moscow, Nauka, 1987 (in Russian).

[20] T.Yang, Y.Yang. A Non-Iterative Reconstruction Algorithm for the Acoustic Inverse Boundary Value Problem. arXiv:2009.00641v1 [math.AP] 1 Sep 2020. 